\title{
Diabetic myonecrosis mimicking acute compartment syndrome of the thigh
}

\begin{abstract}
Diabetic myonecrosis is a very uncommon complication of long-standing diabetes. The presentation is well characterized and management is simple. A 36-year-old lady with a long-standing history of poorly controlled type- 1 diabetes was referred by the renal team with acute pain and swelling of the thigh. The patient had clinical findings of compartment syndrome, however the inflammatory markers and CK were marginally elevated which is not common in compartment syndrome. On two previous admissions the patient was subjected to surgical decompression of the thigh for possible acute compartment syndrome secondary to pyomyositis. On both occasions histology showed sterile non-specific muscle necrosis. An MRI scan performed demonstrated skin thickening and diffuse edema within the subcutaneous tissues and the muscles of the thigh. Given the patient's presentation, previous presentations and subsequent investigations, a diagnosis of diabetic myonecrosis was made and the patient was treated conservatively. The patient recovered uneventfully at 3-weeks, unlike the previous two admissions when she stayed in hospital for 10-12 weeks and because of which a provisional renal transplant date was postponed following the second of the two previous admissions. This report highlights that in patients with long standing diabetes, acute swelling of the limbs should be carefully evaluated for diabetic myonecrosis. Surgical treatment increases morbidity and prolongs recovery in this condition.
\end{abstract}

Keywords: Diabetes, Compartment Syndrome, Myonecrosis
Volume 8 Issue 4 - 2017

\author{
Mohamad Hachem, John Black, Jonathan \\ Compson, Karthik Karuppaiah \\ Department of Trauma and Orthopaedics, Kings College \\ University Hospital, United Kingdom
}

Correspondence: Mohamad Hachem, 30 A Chiswick High Road, Chiswick, London W4 ITE, UK, Tel 448000000000 Email mohamad.hachem@nhs.net

Received: May 18, 2017 | Published: July 10, 2017

\section{Introduction}

Diabetic myonecrosis is an underreported complication of longstanding, poorly controlled diabetes mellitus. ${ }^{1}$ It affects both type 1 and type 2 diabetic patients, is usually self-limiting and responds well to conservative management. ${ }^{1-4}$ It commonly affects the lower limb with thigh muscles being most commonly involved. ${ }^{1,3}$ The disease commonly affects women (61.5\% of all cases), type 1 diabetes patients (59\% of all cases) and patients with long-standing diabetes (mean duration of disease 14.3 years). ${ }^{2}$ Although it most commonly involves a single limb, bilateral involvement has been described in $8.4 \%$ of cases. ${ }^{2}$ Since the condition presents with characteristic symptoms of compartment syndrome,,$^{1-3}$ it can easily be misdiagnosed and treated surgically. Operative intervention increases morbidity and prolongs recovery of the patient. ${ }^{1-3,5}$ We report a case of diabetic myonecrosis presenting with symptoms of compartment syndrome and its management.

\section{Case report}

A 36 year old female was referred to us by the renal team with a possible spontaneous compartment syndrome of the left thigh. The patient had acute onset of severe pain and swelling of the thigh and was unable to weight bear or move the knee. Her analgesic requirements had increased suddenly. There was no history of trauma, fever, chills, rash or insect bites. On examination, the left thigh was tense and swollen, with mild erythema of the overlying skin. The swollen thigh was warm and tender to touch, but there was no obvious blistering or demarcation. Her distal neurovascular status was intact. She was unable to move her knee or perform a straight leg raise due to severe pain in the thigh.

She was known to have type 1 diabetes with diabetic nephropathy chronic kidney disease stage three (CKD III), peripheral neuropathy, and diabetic gastroparesis for which she was on treatment. Her medical history also included coronary artery disease, hypertension, hypothyroidism and intermittent depression.
Her diabetes was poorly controlled on insulin, with her most recent HBA1c being $84 \mathrm{mmol} / \mathrm{mol}(21-42 \mathrm{mmol} / \mathrm{mol})$. She had a normal White cell count with CRP of $159.1 \mathrm{mg} / \mathrm{L}(<5 \mathrm{mg} / \mathrm{L})$ and CK $426 \mathrm{IU} / \mathrm{L}(<150 \mathrm{IU} / \mathrm{L})$. A Doppler scan was negative for DVT and MRI (Figures 1 \& 2) showed left thigh muscle oedema and necrosis affecting the anterior compartment. A possibility of pyomyonecrosis was suggested. To rule out the same, an ultrasound guided biopsy was performed. The aspirate revealed no growth from microbiology and histology revealed fiber atrophy, very few macrophages in the perimyosial connective tissue, and more importantly, thickened and hyalinised walls of small endomyosial and perimyosial capillaries with evidence of necrotic fibers. These findings are highly suggestive of necrotizing myopathy. With pipestem capillaries and minimal cellular infiltration, the possibility of an infective aetiology was ruled out.

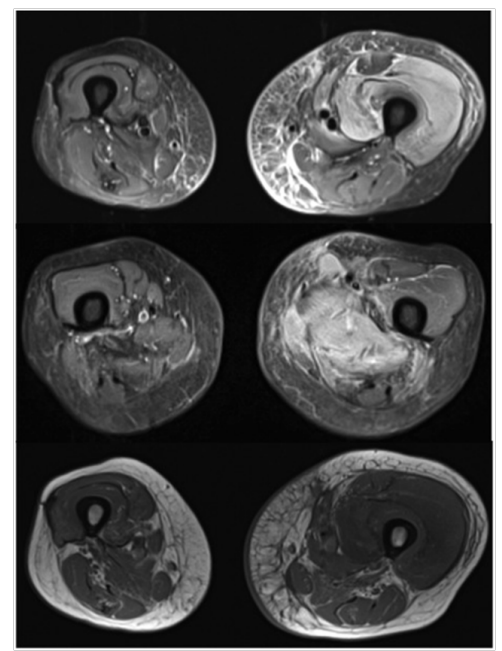

Figure I TI and T2 weighted cross sectional MRI of the both thigh showing increased signal intensity, skin and muscle edema affecting the anterior compartment of the left thigh. 


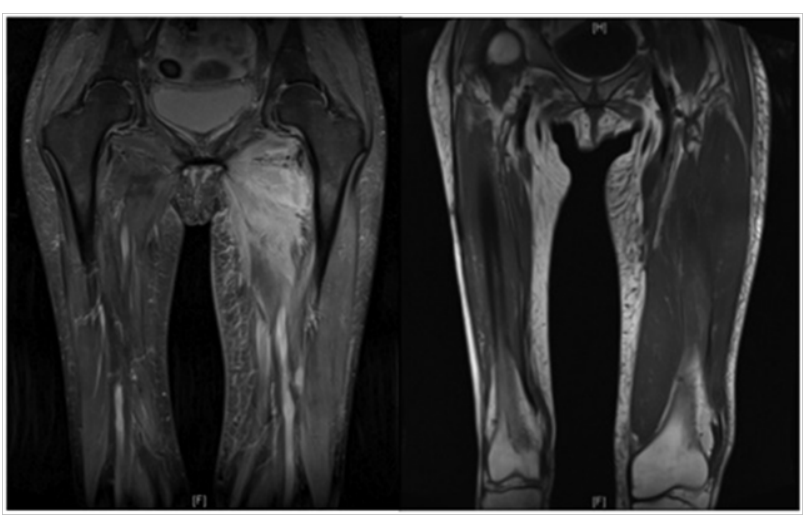

Figure $2 \mathrm{TI}$ and 2 weighted coronal MRI of the both thigh showing increased signal intensity and muscle edema affecting the anterior compartment of the left thigh.

The patient had two admissions in the past with similar symptoms. On the first occasion (18 months prior to current admission), she was treated with a fasciotomy for suspected compartment syndrome and surgical drainage of fluid due to possible pyomyonecrosis in the anterior compartment of the right (contralateral) thigh. The aspirate did not culture a causative organism. Continued treatment with antibiotics and analgesics made little difference to her symptoms, which actually worsened after surgery. Her wound was extremely slow to heal, and contributed to a prolonged inpatient stay of about ten to twelve weeks. On her second admission (six months prior to current admission) she presented with similar symptoms in the left thigh. She again had surgical decompression and debridement. Histology was suggestive of a necrotising myopathy of non-infective aetiology and the patient had a prolonged recovery of 10-12 weeks.

On this latest admission, she presented with similar findings but in light of her clinical findings, recent investigation results and her past history, a diagnosis of diabetic myonecrosis was made and the patient was treated conservatively with optimisation of glycemic control, rest and analgesics. The swelling gradually abated, as did the pain. The CRP and CK came down to 100 and 40 respectively, at the end of first week. The patient was discharged home after 3-4 weeks with a walking frame. This was in contrast to a lengthy stay following her previous admissions that involved surgical intervention and a prolonged period of hospitalisation. The patient made a complete recovery and was mobilizing full weight bearing at six weeks and was asymptomatic at six month follow up.

\section{Discussion}

Diabetic myonecrosis is an uncommon complication associated with diabetes mellitus. Though this condition was first reported in $1965,{ }^{6}$ awareness amongst surgical healthcare professionals remains low. ${ }^{1-3,5,7,8}$ Published studies discuss the difficulties in diagnosis, and also how this condition has a tendency to be under recognised and therefore sub-optimally managed. ${ }^{1-3,9}$ The pathophysiology of this condition is not yet fully understood, however, atheromatous emboli, thrombo-occlusive disorders and arteriosclerosis have been implicated. ${ }^{2,8}$ This disease is more common in females, in type 1 diabetes and in long standing diabetes, as is the case with our patient. ${ }^{8}$

The most commonly affected muscles are quadriceps (60-65\%), hip adductors $(13 \%)$, hamstrings $(8 \%)$, and hip flexors $(2 \%){ }^{10}$ However, it can affect the muscles of the leg compartments or rarer still, the upper limb muscles., ${ }^{2,4}$ As this is a diagnosis of exclusion, other common conditions mimicking compartment syndrome (deep venous thrombosis, thrombophlebitis, haematoma, fracture, pyomyositis, necrotising, fasciitis, myositis or dermatomyositis, abscess, cellulitis, connective tissue tumor) have to be excluded..$^{1-3,8}$ Though most patients have long standing diabetes with poor glycemic status and microvascular complications, ${ }^{2}$ rarely it has been reported as the first manifestation of diabetes mellitus. ${ }^{11}$

Though sonography and CT scan have been utilized for diagnosis, MRI is the investigation of choice. ${ }^{1,2,12}$ MRI typically shows diffuse muscle oedema with necrosis and increased signal in T2-weighted, inversion-recovery, and gadolinium enhanced images and isointense or hypointense areas on T1- weighted images..$^{13}$ The combination of this with normal or slightly elevated inflammatory markers and CPK are highly suggestive of diabetic myonecrosis. Muscle biopsy is recommended in doubtful cases, not as a routine due to potential complications and delayed recovery. ${ }^{2}$

Diabetic myonecrosis is a self-limiting condition and can be easily managed by simple observation and supportive therapy. ${ }^{2}$ Studies suggest that patients who had surgery had an average recovery period of 13 weeks when compared to 5.5 weeks for those treated conservatively. ${ }^{2,3}$ The short-term prognosis of this condition is good. However, patients who present with this condition have an increased 5-year mortality rate, regardless of other diabetes-related co-morbidities..$^{14} \mathrm{~A}$ recurrence rate of $47.82 \%$ has been reported, in the same or the other limb (as in our case). ${ }^{2}$

Spontaneous compartment syndrome following DM is extremely rare and very few cases have been reported in the literature affecting the leg but none in the thigh muscles. ${ }^{15-19}$ In all the cases following fasciotomy, patients ended up with either foot drop or weak dorsiflexion and prolonged rehabilitation times. To reduce morbidity, one author suggests a single incision fasciotomy. ${ }^{19}$

This case highlights the likely recurrence of this condition and how diabetic myonecrosis can be misdiagnosed. This can lead to unnecessary surgical interventions, which in our patient previously resulted in exacerbated symptoms and prolonged hospital stays. Simultaneously, one needs to be cautious that compartment syndrome is not missed. All patients should be thoroughly evaluated by means of history, baseline blood investigations, (especially for inflammatory markers and CK) and with MRI before a diagnosis of diabetic myonecrosis can be made.

\section{Conclusion}

Diabetic myonecrosis should be considered in patients presenting with symptoms of atraumatic acute compartment syndrome and longstanding diabetes. It is a diagnosis of exclusion and is best managed conservatively as surgical treatment delays recovery and increases morbidity.

\section{Conflicts of Interest}

There is no conflict of interest related to the above manuscript.

\section{Acknowledgments}

None.

\section{References}

1. Choudhury BK, Saikia UK, Sarma D, et al. Diabetic myonecrosis: An underreported complication of diabetes mellitus. Indian J Endocrinol Metab. 2011;15(Suppl 1):S58-S61.

2. Trujillo-Santos AJ. Diabetic muscle infarction: An undiagnosed complication of long standing diabetes. Diabetes Care. 2003;26:211-215. 
3. Kapur S, McKendry RJ. Treatment and outcome of diabetic muscle infarction. J Clin Rheumatol. 2005;11(1):8-12.

4. Uçan H, Alemdaroğlu E, Yoldaş TK, et al. Diabetic muscular infarct: an unusual cause of extremity pain and dysfunction. Rheumatol Int 2012;32(2):525-528.

5. Bhasin R, Ghobrial I. Diabetic myonecrosis: a diagnostic challenge in patients with long-standing diabetes. J Community Hosp Intern Med Perspect. 2013;3(1).

6. Angervall L, Stener B. Tumoriform focal muscle degeneration in two diabetic patients. Diabetologia. 1965;1:39-42.

7. Glauser SR, Glauser J, Hatem SF. Diabetic muscle infarction: rare complication of advanced diabetes mellitus. Emerg Radiol. 2008;15(1):61-65.

8. Bunch TJ, Birskovich LM, Elken PW. Diabetic myonecrosis in a previously helathy women and review of a 25 year Mayo clinic experience. Endocrinology practice. 2002;8:343-346.

9. Martínez JH, Torres O, Mangual García MM, et al. Diabetic myonecrosis: an atypical presentation. Case Rep Endocrinol. 2013;2013:190962.

10. Rashidi A, Bahrani O. Diabetic myonecrosis of the thigh. J Clin Endocrinol Metab. 2011;96(8):2310-2311.

11. Khan S, Al-Riyami D, Al-Ghoulani M. An under recognized complication: Diabetic myonecrosis. Oman Med J. 2009;24(3):228-230.
12. Delaney-Sathy LO, Fessel DP, Jacobson JA, et al. Sonography of diabetic muscle infarction with MR imaging, CT and pathologic correlation. AJR Am J Roentgenol. 2000;174(1):165-169.

13. Jeleinek JS, Murphy MD, Aboulafia AJ, et al. Muscle infarction in patients with diabetes mellitus: MRI images finding. Radiology. 1999;211(1):241-247.

14. Hoyt JR, Wittich CM. Diabetic myonecrosis. J Clin Endo Meta. 2008;93(10):3690.

15. Abisi S, Yong YP, Beech A, et al. Spontaneous sequential compartment syndrome of the lower limbs. Vasc Endovascular Surg. 2013;47(7):566-568.

16. Flamini S, Zoccali C, Persi E, et al. Spontaneous compartment syndrome in a patient with diabetes and statin administration: a case report. $J$ Orthop Traumatol. 2008;9(2):101-103.

17. Woolley SL, Smith DR. Acute compartment syndrome secondary to diabetic muscle infarction: case report and literature review. Eur $J$ Emerg Med. 2003;13(2):113-116.

18. Jose RM, Viswanathan N, Aldlyami E, et al. A spontaneous compartment syndrome in a patient with diabetes. J Bone Joint Surg Br. 2004;86(7):1068-1070.

19. Pamoukian VN, Rubino F, Iraci JC. Review and case report of idiopathic lower extremity compartment syndrome and its treatment in diabetic patients. Diabetes Metab. 2000;26(6):489-492. 